\title{
Follicular fluid cerebellin and betatrophin regulate the metabolic functions of growing follicles in polycystic ovary syndrome
}

\author{
Aynur Adeviye Ersahin ${ }^{1}$, Mustafa Acet ${ }^{2}$, Suat Suphan Ersahin ${ }^{3}$, Tuba Acet ${ }^{4}$, Meltem Yardim ${ }^{5}$, Omer Kenanoglu ${ }^{5}$, Suleyman Aydin \\ 'Department of Obstetrics and Gynecology, Bahcesehir University School of Medicine, Istanbul; ${ }^{2}$ Department of Obstetrics and Gynecology, Medipol \\ University School of Medicine, Istanbul; ${ }^{3}$ Department of Obstetrics and Gynecology, Kemerburgaz University School of Medicine, Istanbul; \\ ${ }^{4}$ Department of Obstetrics and Gynecology, Medicine Hospital, Istanbul; ${ }^{5}$ Department of Medical Biochemistry (Firat Hormones Research Group), Firat \\ University School of Medicine, Elazig, Turkey
}

Objective: The aim of this study was to assess the changes of follicular fluid (FF) and serum levels of cerebellin precursor protein 1 (cbln1) and betatrophin in patients with polycystic ovary syndrome (PCOS) undergoing in vitro fertilization/intracytoplasmic sperm injection (IVF/ICSI) with a gonadotropin-releasing hormone $(\mathrm{GnRH})$ antagonist protocol.

Methods: Twenty infertile women with PCOS and 20 control women diagnosed as poor responders undergoing ovarian stimulation with a $\mathrm{GnRH}$ antagonist were included. Blood samples were obtained during ovum pick-up. Follicular fluid from a dominant follicle was collected from the subjects. Using enzyme-linked immunosorbent assays, FF and serum levels of $\mathrm{cbln} 1$ and betatrophin were measured in both groups of participants. Metabolic and hormonal parameters were also determined and correlated with each other.

Results: Both groups of women had similar serum and FF betatrophin levels $(55.0 \pm 8.9 \mathrm{ng} / \mathrm{mL}$ vs. $53.1 \pm 10.3 \mathrm{ng} / \mathrm{mL}, p=0.11)$. The serum and FF betatrophin levels of poor responders were found to be similar ( $49.9 \pm 5.9 \mathrm{ng} / \mathrm{mL}$ vs. $48.9 \pm 10.7 \mathrm{ng} / \mathrm{mL}, p=0.22)$. Conversely, the FF cbln1 levels of PCOS women were found to be significantly higher than the serum cbln1 levels $(589.1 \pm 147.6 \mathrm{ng} / \mathrm{L} \mathrm{vs.} 531.7 \pm 74.3 \mathrm{ng} / \mathrm{L}, p<0.02)$. The FF cbln1 levels of control participants without PCOS were significantly higher than their serum cbln1 levels $(599.3 \pm 211.5 \mathrm{ng} / \mathrm{L}$ vs. $525.3 \pm 87.0$ $\mathrm{ng} / \mathrm{L}, p=0.01$ ). Positive correlations were detected among body mass index, insulin resistance, serum insulin, total testosterone, and betatrophin levels in the PCOS group.

Conclusion: Follicular fluid betatrophin and cbln1 concentrations may play a pivotal role on follicular growth in PCOS subjects undergoing IVF/ ICSI with an antagonist protocol.

Keywords: Antagonist protocol; Betatrophin; Cerebellin; Follicular fluid; Intracytoplasmic sperm injection; In vitro fertilization; Polycystic ovary syndrome

\section{Introduction}

Polycystic ovary syndrome (PCOS), hyperandrogenism, oligo-an-

Received: Aug 13, 2016 · Revised: Jan 9, 2017 · Accepted: Feb 2, 2017

Corresponding author: Aynur Adeviye Ersahin

Department of Obstetrics and Gynecology, Bahcesehir University School of Medicine, Osmanpaşa Mektebi Street 4-6, 34349 Beşiktaş, Istanbul, Turkey Tel: +90-2164684588 Fax:+90-4242379138 E-mail:aynur.ersahin@hotmail.com

This is an Open Access article distributed under the terms of the Creative Commons Attribution Non-Commercial License (http://creativecommons.org/licenses/by-nc/4.0/) which permits unrestricted non-commercial use, distribution, and reproduction in any medium, provided the original work is properly cited. ovulation, and associated insulin resistance affect up to one in five reproductive-aged women $[1,2]$. The identification of more peptides with a potential role in the etiology of PCOS has improved our knowledge about the close interaction between follicular development and peptides. Even independently of PCOS, complex interactions occur among peptides, hypothalamo-pituitary neurons, and follicle development. This observation warrants the identification of central and peripheral peptides in conditions of impaired metabolic status, with special attention focused on PCOS.

Relationships between the peptides involved in insulin metabolism 
and follicular development have been reported. Inadequate crosstalk among peptides, peripheral adipose tissues, and specific brain regions in PCOS may have a negative impact on normal follicular development. Concordantly, high intrafollicular leptin in PCOS subjects undergoing in vitro fertilization/intracytoplasmic sperm injection (IVF/ICSI) has been shown by Fedorcsak et al. [3]. Moreover, both the serum and follicular fluid (FF) levels of irisin peptide were found to be negatively correlated with insulin resistance in PCOS [4]. Alteration in the circulating levels of any peptide can cause defective secretion of gonadotropin-releasing hormone and gonadotropins in patients with PCOS $[5,6]$. Peptides may have also a direct impact on reproductive tissues [7]. In line with these findings, metabolic disturbances in PCOS subjects may adversely affect the secretion of peripheral or central peptides, and vice versa. We therefore decided to investigate the circulating levels of 1 central and 1 peripheral peptide in patients with PCOS: cerebellin precursor protein 1 (cbln1) and betatrophin. Although most PCOS subjects have high serum levels of different hormones and peptides, the impact of betatrophin and cbln 1 on the biological parameters of PCOS subjects remains elusive. Both peptides may play a role in the occurrence of PCOS by affecting the developmental potential of oocytes and insulin metabolism.

Betatrophin (also known as ANGPTL8) is a recently identified peptide that is synthesized in the liver as well as white and brown adipose tissue $[8,9]$. It regulates pancreatic beta cell function and lipid production [10]. Circulating levels of betatrophin are altered by both physiological and pathological conditions, such as metabolic disorders and obesity [11-13]. In fact, close associations among circulating betatrophin levels, type 1 diabetes, and PCOS have been reported [11-13]. Likewise, high serum betatrophin levels were reported in patients with obesity or insulin resistance [14-16]. Pancreatic beta cell proliferation can be induced by insulin resistance. In line with this, a recent study showed that betatrophin stimulated beta cell proliferation in the presence of insulin resistance [8]. Since PCOS is characterized by insulin resistance, betatrophin levels may change in both the serum and in other biological compartments, including the FF of developing oocytes.

$\mathrm{Cbln} 1$ is a novel central peptide that is essential for normal synaptic architecture and function in the cerebellum. In addition to its central action, cbln 1 has been found to be active in the adrenal cortex, pituitary, heart, kidney, and adrenal tumors $[17,18]$. Four different isoforms have been noted, which are termed cbln1 through cbln4, and they are characterized by a conserved C1q domain [19]. Cbln1, cbln2, and cbln4 are released as glycoproteins, whereas cbln3 remains within the endoplasmic reticulum [19]. Although much is known about the action of cbln1, little is known concerning the other family members. They are located mainly in the cerebellum and function there. Moreover, little is known about the function of $\mathrm{cbln} 1 \mathrm{in}$ periph- eral tissues. It binds to the so-called orphan glutamate receptor GluR22 on Purkinje cells [20]. By inducing adenylate cyclase, protein kinase $A$, and steroid hormone secretion, cbln1 induces catecholamine release $[21,22]$. Nevertheless, the precise role of cbln1 in reproductive or metabolic disorders, including PCOS, is not clear. Knockout mice models for cerebellin are fertile and have no anatomical defects. A recent study by Burnet et al. [23] showed cerebellin-like immunoreactivity in the rat pancreas and in guinea pigs. Likewise, Strowski et al. [24] demonstrated the expression of cbln1 and cbln3 in the pancreas of rats. They also noted evidence that cerebellin exerts insulinostatic activity in vivo and in vitro.

The unbalanced secretion of peripheral and central peptides involved in insulin metabolism led us to suppose that both serum and FF levels of cbln 1 and betatrophin may be altered in PCOS. Although several investigations have analyzed the serum and tissue levels of betatrophin and cbln1 in subjects with metabolic disorders, their levels in the serum and FF of PCOS subjects have not been analyzed yet. This study was therefore planned to find a logical answer to two questions. The first aim was to investigate whether FF cbln1 and betatrophin production changed in infertile PCOS subjects undergoing IVF/ICSI. The second aim was to investigate the relationships between the measured levels of these peptides in serum and the FF of PCOS subjects and insulin resistance. Possible associations among serum and FF cbln1, betatrophin levels, demographic variables, and laboratory parameters were also analyzed.

\section{Methods}

In order to determine the minimum number of subjects that needed to be enrolled in this study to ensure sufficient statistical power, a sample size calculation was carried out before the study. The probability of a type I error (a) (i.e., finding a difference although a difference does not exist) was calculated. We used an alpha cut-off of $5 \%$ (0.05). Serum and FF cerebellin and betatrophin concentrations were considered to be significant at a type I error cut-off of 0.05 with a power of 0.85 . Together, 20 patients in the study and control groups were required to ensure adequate study power, so 20 patients with the diagnosis of PCOS and 20 control cases diagnosed as poor responders (PORs) were included in the study. Participants with PCOS and control participants without PCOS were matched according to their body mass index (BMI). Participants in both groups met the inclusion and exclusion criteria for PCOS and POR. A participant was diagnosed with PCOS if she had two of the following three findings: (1) oligo-anovulation, (2) either clinical or biochemical hyperandrogenism, and (3) an ultrasonographic diagnosis of polycystic ovaries [25]. Participants in the control group were diagnosed as PORs when at least two of the following three findings were present: (1) age $\geq 40$ 
years, (2) a previous history of poor ovarian response (an oocyte retrieval of $<$ three oocytes with a conventional induction method), or (3) a decline in ovarian reserve $(<5-7$ antral follicles in ultrasonography or serum anti-Müllerian hormone levels of $<0.5-1.1 \mathrm{ng} / \mathrm{mL}$ ). Women with the diagnosis of premature ovarian aging and severe endometriosis were not included. Women with a single ovary, a previous history of ovarian cystectomy, a history of chronic smoking, or previous chemotherapy and/or radiotherapy treatment were excluded.

A blood test was performed on the second to fifth day of progesterone-induced bleeding in patients with anovulatory PCOS and on day 3 among ovulatory PCOS subjects and PORs. Fasting blood samples were used for biochemical and hormonal evaluations. For insulin resistance, the homeostasis model assessment of insulin resistance (HOMA-IR) formula was used: fasting serum insulin mlU/L $\times$ fasting glucose (mg/dL)/405 [26]. This study was approved by the local investigation and ethics committee of Istanbul Medipol University Hospital.

\section{FF and serum cbln 1 and betatrophin measurements}

Both the serum and FF samples used in this study were the same specimens collected for the measurement of irisin in our previous study [4]. When reviewing the literature, no study was found that investigated FF and serum betatrophin and cbln1 levels in PCOS subjects undergoing an antagonist protocol. Since these 2 peptides have been recently identified, we decided to measure their levels in the same samples and the same patients undergoing IVF/ICSI. The steps of the controlled ovarian stimulation protocol and sample collection can be found elsewhere [4]. As noted previously, venous blood and FF samples were collected during ovum pick-up in both groups of participants [4]. Participants with other endocrine disorders or who took any medication were excluded. Women with bloody FF during ovum pick-up were also excluded. After the removal of the cumulusoocyte complex, the remaining follicular contents were put into tubes and centrifuged at 3,000 $\mathrm{g}$ for 10 minutes and were stored at $-80^{\circ} \mathrm{C}$.

Both serum and FF betatrophin and cbln1 concentrations were quantitatively measured using enzyme-linked immunosorbent assays (ELISA) kits according to the manufacturer's instructions (human betatrophin ELISA kit, human cerebellin ELISA kit, REF: E20160202002; Rel Assay Diagnostics, Gaziantep, Turkey). This assay was used to measure serum and FF cbln1 and betatrophin levels in both groups of participants. Significant cross-reactivity or interference affecting the measurements was not detected. The sensitivity of the assays for betatrophin and cbln1 was $0.23 \mathrm{ng} / \mathrm{mL}$ and $2.34 \mathrm{ng} / \mathrm{L}$, respectively. The inter- and intra-assay coefficients of variation for both peptides were found to smaller than $10 \%$ and $12 \%$, respectively. The assay range for betatrophin was 0.5 to $100 \mathrm{ng} / \mathrm{mL}$, and it was 5 to 1,500 $\mathrm{ng} / \mathrm{L}$ for cbln1. The method validation for both biological samples was carried out as defined by Aydin [27]. FF and serum betatrophin and cbln1 levels were measured with a ChroMate P4300 microplate reader (Awareness Technology Inc., Palm City, FL, USA).

\section{Statistical analysis}

For the statistical analysis of all demographic and laboratory findings from both groups of participants, SPSS ver. 21.0 (IBM Corp., Armonk, NY, USA) was used. The normality of individual group parameters was assessed with the one-sample Kolmogorov-Smirnov $Z$ test. They were found to be abnormally distributed in both groups. The Mann-Whitney $U$ test and Wilcoxon test were used for comparisons of PCOS and control subjects. Correlations among the serum and FF levels of betatrophin, cbln1, retrieved oocytes, and serum levels of estradiol on human chorionic gonadotropin (hCG) day, clinical pregnancy rates, and other parameters were evaluated using the Spearman correlation method. Data were given as mean \pm standard deviation. The $p$-values $<0.05$ were considered to indicate statistical significance.

\section{Results}

The laboratory characteristics of both groups, including serum and FF cbln 1 and betatrophin levels, are presented in Table 1. Women with PCOS were younger than those in the control group. The infertility duration of the PORs was longer than those in the PCOS group. Although the BMI of PCOS patients was higher, this did not reach statistical significance. Although the PCOS subjects had high insulin, total testosterone, and HOMA-IR levels, their estradiol and LH concentrations were similar to the control group. The basal FSH levels that were measured on day 3 among the POR subjects were found to be significantly higher than those in the PCOS subjects. Both the number of retrieved oocytes during ovum pick-up (20 vs. 3) and the clinical pregnancy rate $(75 \%$ vs. $35 \%)$ were significantly higher in the PCOS group than among the PORs. Likewise, the serum levels of es-

Table 1. Serum and FF levels of betatrophin and cbln1 in each group

\begin{tabular}{lccl}
\hline Variable & PCOS & Poor responder & $p$-value \\
\hline Serum betatrophin $(\mathrm{ng} / \mathrm{mL})^{\mathrm{a})}$ & $55.0 \pm 8.9$ & $49.9 \pm 5.9$ & 0.054 \\
FF betatrophin $(\mathrm{ng} / \mathrm{mL})^{\mathrm{b})}$ & $53.1 \pm 10.3$ & $48.9 \pm 10.7$ & 0.11 \\
Serum cbln1 $(\mathrm{ng} / \mathrm{L})^{\mathrm{c})}$ & $531.7 \pm 74.3$ & $525.3 \pm 87.0$ & 0.74 \\
FF cbln1 $(\mathrm{ng} / \mathrm{L})^{\mathrm{d})}$ & $589.1 \pm 147.6$ & $599.3 \pm 211.5$ & 0.74 \\
$p$-value & & & \\
a) vs. b) & 0.11 & 0.22 & - \\
c) vs. d) & 0.02 & 0.01 & - \\
\hline
\end{tabular}

Values are presented as mean \pm standard deviation.

FF, follicular fluid; cbln1, cerebellin precursor protein 1; PCOS, polycystic ovary syndrome. 
tradiol in PCOS subjects on the day of hCG administration were found to be significantly higher than those in the control group.

\section{Serum and FF betatrophin and cbln1 levels in PCOS patients}

Serum and FF betatrophin concentrations in PCOS subjects were similar $(55.0 \pm 8.9 \mathrm{ng} / \mathrm{mL}$ vs. $53.1 \pm 10.3 \mathrm{ng} / \mathrm{mL}, p=0.11)$. A significant difference between $\mathrm{FF}$ and serum cbln1 levels in PCOS subjects was detected ( $589.1 \pm 147.6 \mathrm{ng} / \mathrm{L}$ vs. $531.7 \pm 74.3 \mathrm{ng} / \mathrm{L}, p=0.02)$. FF cbln1 levels were found to be significantly higher than serum levels cbln1 in PCOS subjects. The serum betatrophin concentrations of women with PCOS were found to be similar to the serum betatrophin levels of PORs $(55.0 \pm 8.9 \mathrm{ng} / \mathrm{mL}$ vs. $49.9 \pm 5.9 \mathrm{ng} / \mathrm{mL}, p=0.054)$. Likewise, a significant difference was not detected between the PCOS and POR groups regarding FF betatrophin levels ( $53.1 \pm 10.3$ $\mathrm{ng} / \mathrm{mL}$ vs. $48.9 \pm 10.7 \mathrm{ng} / \mathrm{mL}, p=0.11)$. When compared to the control group, PCOS patients had similar serum cbln1 $(531.7 \pm 74.3 \mathrm{ng} / \mathrm{L}$ vs. $525.3 \pm 87.0 \mathrm{ng} / \mathrm{L}, p=0.74)$ and FF cbln1 levels $(589.1 \pm 147.6 \mathrm{ng} /$ L vs. $599.3 \pm 211.5 \mathrm{ng} / \mathrm{L}, p<0.74$ ).

Table 2 shows a correlation analysis of serum and FF betatrophin and cbln1 levels with each other and with laboratory and demographic parameters. Spearman correlation analysis showed no significant association between FF and serum betatrophin levels in PCOS subjects. Likewise, we did not detect any correlation between FF and serum cbln1 levels. However, a positive correlation was detected between serum betatrophin and total testosterone levels $(r=0.53$, $p<0.01)$ in PCOS subjects. Positive correlations were also detected among HOMA-IR, serum insulin, and betatrophin levels $(r=0.47$, $p=0.03 ; r=0.30, p=0.03$, respectively). Moreover, we found a positive correlation between serum betatrophin and BMI in the PCOS group $(r=0.29, p=0.04)$. FF betatrophin levels were also found to be positively correlated with BMI, HOMA-IR, and serum insulin levels in PCOS subjects. A significant correlation was not detected between FF betatrophin and serum levels of testosterone. Serum levels of betatrophin were negatively correlated with FF cbln $1(r=-0.386$,

Table 2. Spearman correlation coefficients $(r)$ among cbln1, betatrophin, and clinical and hormonal parameters in subjects with PCOS

\begin{tabular}{lcccccc}
\hline \multirow{2}{*}{ Independent variable } & \multicolumn{2}{c}{ Serum betatrophin } & & \multicolumn{2}{c}{ FF betatrophin } \\
\cline { 2 - 3 } \cline { 5 - 6 } & \multicolumn{1}{c}{$r$} & $p$-value & & $r$ & $p$-value \\
\hline Serum cbln1 & 0.130 & 0.47 & & -0.224 & 0.24 \\
FF cbln1 & -0.386 & 0.035 & & 0.346 & 0.04 \\
BMl & 0.29 & 0.04 & & 0.47 & 0.03 \\
HOMA-IR & 0.47 & 0.03 & & 0.36 & 0.01 \\
Testosterone & 0.53 & 0.01 & & 0.51 & 0.43 \\
Insulin & 0.3 & 0.03 & & 0.47 & 0.02 \\
\hline
\end{tabular}

cbln1, cerebellin precursor protein 1; PCOS, polycystic ovary syndrome; FF, follicular fluid; BMI, body mass index; HOMA-IR, homeostasis model assessment of insulin resistance. $p=0.035)$ in PCOS patients. FF betatrophin levels of PCOS subjects were positively correlated with FF cbln1 levels $(r=0.346, p=0.04)$. Since PCOS subjects had a high oocyte retrieval number $(>20)$ compared to the control subjects $(<3)$, a correlation analysis between the measured peptide levels and the number of total oocytes was not performed. We did not find any significant associations among the serum levels of estradiol on the hCG day, clinical pregnancy rates, and the serum and FF levels of betatrophin in either group of subjects. However, a positive but insignificant correlation was detected between FF cbln1 and estradiol levels on the day of hCG administration. Likewise, no correlations were detected between serum betatrophin and cbln 1 levels and circulating estradiol levels on the day of hCG administration.

\section{Serum and $\mathrm{FF}$ betatrophin and cbln1 levels in PORs}

The serum and FF betatrophin levels of POR subjects were found to be similar $(49.9 \pm 5.9 \mathrm{ng} / \mathrm{mL}$ vs. $48.9 \pm 10.7 \mathrm{ng} / \mathrm{mL}, p=0.22)$. Similarly to $\mathrm{PCOS}$ patients, the FF cbln1 levels of PORs were significantly higher than their serum cbln1 levels $(599.3 \pm 211.5 \mathrm{ng} / \mathrm{L}$ vs. $525.3 \pm 87.0$ $\mathrm{ng} / \mathrm{L}, p<0.01$ ). Neither a negative nor positive correlation was found between serum and FF betatrophin and cbln1 levels in the POR group. No correlations were detected among the demographic variables, laboratory parameters, and measured peptide levels in the serum and FF of the control subjects.

\section{Discussion}

Although the underlying cause of defective folliculogenesis is not well understood in PCOS, there is some evidence that abnormalities in some specific peptides may be associated with this condition [2]. Indeed, by reducing the rates of pregnancy and fertilization in rats, obesity secondary to metabolic disorders can adversely affect the outcome of IVF/ICSI [28-30]. In line with this, fewer oocytes or impaired embryo quality and an increased risk of spontaneous abortion have been reported in women with PCOS [30]. The failed reproductive outcomes that may be seen in obese patients may be mediated by insulin or by other peptides related to insulin metabolism [31]. The impact of insulin resistance and hyperinsulinemia on follicle development in women undergoing IVF/ICSI was reported in a study conducted by Fedorcsak et al. [32]. They showed that women with PCOS and insulin resistance required a higher follicle-stimulating hormone dose to induce ovulation. They also noted that the presence of insulin resistance led to a decline in circulating estradiol levels compared to women with normal insulin sensitivity. A study by Stadtmauer et al. [33] was conducted on PCOS patients resistant to clomifene who underwent IVF/ICSI. The authors showed that the addition of metformin led to the pick-up of more mature oocytes, a 
higher fertilization rate, and a higher pregnancy rate. This is the first study measuring FF cbln 1 and betatrophin levels in PCOS patients undergoing IVF/ICSI with an antagonist protocol. Due to significant cross-reactivity or interference between measured laboratory parameters, demographic variables, and the levels of any peptide in biological fluids, evaluating the concentration of peptides is associated with challenges in terms of the laboratory method used. We were able to detect cbln 1 and betatrophin in the FF of PCOS subjects and control cases without PCOS. Our study clearly demonstrated that the serum levels of cbln1 in PCOS subjects were significantly lower than the FF cbln1 levels. Likewise, the control subjects without PCOS showed lower serum cbln1 levels than FF cbln1 levels. Nevertheless, no significant correlation was detected between serum and FF cbln1 levels, suggesting the independent regulation of cbln1 in the growing follicle.

The follicular cells of the growing oocytes in PCOS subjects may produce cbln1 to regulate steroid hormone production. Nevertheless, the observation of increased levels of FF cbln1 in control cases without PCOS weakens our hypothesis. Several explanations may exist for the increase in FF cbln1 levels in both groups of subjects. PCOS may not involve the increased synthesis of cbln1 as part of the disease process. The high levels of FF cbln1 in control cases without PCOS support this idea. Another explanation of high FF cbln1 concentrations in all participants may be the method used for ovarian stimulation. In the current study, we used an antagonist protocol for ovarian stimulation. Concordantly, the presence of high FF cbln1 levels in both groups of subjects might be secondary to gonadotropins or antagonists that might induce cbln1 production from the cumulus-oocyte complex. Low levels of circulating cbln1 support the critical role of both the drugs of the antagonist protocol and follicle cells. In agreement with this, the positive but insignificant correlation between FF cbln 1 and estradiol levels on the day of hCG administration supports the idea that the follicle plays a pivotal role in producing cbln1. Further support for this stems from the fact that it has been reported that cbln1 enhanced steroid hormone secretion from the adrenal glands [21]. Moreover, the disturbed secretion of some biochemical compounds and hormones in PCOS patients supports our idea [34]. Together, regardless of the presence of PCOS, cbln1 may contribute to follicular development in healthy women and women with metabolic defect. To provide insight into this mystery, studies comparing FF cbln1 levels between subjects undergoing natural-cycle IVF and subjects undergoing an antagonist protocol are required to determine the impact of the induction protocol and of follicular cells on FF cbln1 production.

Although cbln1 induces norepinephrine release from the adrenal glands [21], the precise function of cbln1 in cellular reactions is controversial. A recent study by Strowski et al. [24] showed the local pro- duction of cbln 1 in the pancreas. They also reported that cbln 1 could regulate insulin release through a modulation of cyclic adenosine monophosphate- and calcium-dependent signal transduction in the pancreas. They also noted the presence of cerebellin immunoreactivity close to glucagon- and somatostatin-producing cells. However, cerebellin immunoreactivity was found to be lower in the central part of the pancreas, which contains many insulin-producing beta cells [24]. Significantly increased FF cbln1 levels in both groups of women suggest that the transfer of cbln1 from FF to the circulation was not allowed. If cbln1 was transported from the FF to the circulation by a receptor-mediated system, the release of cbln1 into the circulation would be facilitated by the presence of high levels of FF cbln1. Due to the presence of similar circulating and FF betatrophin levels in PCOS and control subjects, it is logical to think that a bidirectional transition process exists for betatrophin between the serum and the FF. However, we did not find any relationship between the two compartments regarding circulating and FF cbln1 levels. In light of these findings, we propose that the cbln 1 in the FF is mainly locally produced by oocytes or follicle cells, excluding the transport of cbln1 from the circulation into the FF.

The insignificant increase in serum betatrophin levels in PCOS subjects when compared to control cases without PCOS led us to think that serum levels of betatrophin were not changed in PCOS subjects. In contrast to cbln2, the drugs used in the antagonist protocol could not affect betatrophin synthesis in the follicle cells. It is quite likely that the follicular cells of both subjects with and without PCOS may not have the ability to synthesize betatrophin. It is possible that the bidirectional transport of betatrophin may maintain balance between the two compartments. In fact, the high vascularity seen in the follicles of PCOS patients may facilitate this transport. The results of studies investigating serum betatrophin levels in patients with metabolic disorders are controversial. In the present study, positive associations among HOMA-IR, serum insulin, BMI, FF, and serum betatrophin levels were detected in PCOS subjects. Our findings are consistent with the results of Calan et al. [12], who showed that circulating betatrophin levels were positively correlated with insulin resistance [12]. Conversely, a study conducted by Gomez-Ambrosi et al. [15] reported that serum betatrophin levels were not related to insulin sensitivity in subjects with new-onset type 2 diabetes. The positive associations among circulating betatrophin, HOMA-IR, and BMI may suggest that unlike insulin resistance, the bidirectional transport of betatrophin between the serum and the follicular compartment is not compromised in individuals with PCOS. Therefore, we can make the strong suggestion that the impact of betatrophin on folliculogenesis in women undergoing IVF with an antagonist protocol is independent of insulin resistance and the underlying causes of infertility [4].

Increased androgen levels in PCOS subjects may facilitate the bal- 
ance of betatrophin levels between the serum and FF compartments. In line with this, the circulating betatrophin levels of PCOS subjects were found to be positively correlated with increased serum androgen and C-reactive protein levels [12]. Likewise, close associations between serum androgens and low-grade inflammation were detected in PCOS subjects [35]. In good agreement with these findings, positive associations between circulating betatrophin and total testosterone levels were found in the present study. Collectively, we strongly suggest that serum levels of androgens might contribute to the regulation of serum and FF betatrophin concentrations. However, in order to draw a clear conclusion regarding the effect of betatrophin on follicular development in PCOS subjects, immunohistochemical studies evaluating the existence of betatrophin and its receptors in oocytes, mural, and cumulus granulosa cells are necessary.

Although the main causes of PCOS-related subfertility remain to be identified, it is believed that the defective production and secretion of some peptides, including betatrophin and cbln1, can play an important role in the pathogenesis of failed ovulation in PCOS. Unfortunately, neither the production site nor the physiological function of either peptide in terms of follicular development in subjects with metabolic dysfunction are well characterized. In the current investigation, the presence of cbln1 and betatrophin was for the first time established in the FF of subjects with and without PCOS. We clearly showed that FF cbln1 levels were found to be elevated in women with and without PCOS. Significantly increased FF cbln1 levels in both groups of women led us to think that the follicular cells of growing oocytes may synthesize cbln1 to regulate steroid hormone production. The lack of a significant correlation between FF and serum levels of cbln1 suggests the independent regulation of this peptide within the growing follicle. However, the lack of any associations among the cbln1, betatrophin, serum estradiol levels, and clinical pregnancy rates weakens the possibility that these peptides may have a role on follicular development in PCOS. In contrast to cbln1, the presence of positive correlation of HOMA-IR, serum insulin, and $\mathrm{BMI}$ with the serum and FF levels of betatrophin suggests that betatrophin may have a critical impact on follicle growth in women with PCOS undergoing IVF/ICSI with an antagonist protocol. In light of our findings, we concluded that the measurement of circulating cbln1 and betatrophin may support the potential role of both peptides as a peripheral marker for follicular well-being. These 2 peptides may play a role in understanding unknown aspects of follicular development in patients with PCOS undergoing treatment with assisted reproductive technology.

\section{Conflict of interest}

No potential conflict of interest relevant to this article was reported.

\section{References}

1. March WA, Moore VM, Willson KJ, Phillips DI, Norman RJ, Davies MJ. The prevalence of polycystic ovary syndrome in a community sample assessed under contrasting diagnostic criteria. Hum Reprod 2010;25:544-51.

2. Celik O, Sahin I, Celik N, Hascalik S, Keskin L, Ozcan H, et al. Diagnostic potential of serum $\mathrm{N}$-terminal pro-B-type brain natriuretic peptide level in detection of cardiac wall stress in women with polycystic ovary syndrome: a cross-sectional comparison study. Hum Reprod 2007;22:2992-8.

3. Fedorcsak P, Storeng R, Dale PO, Tanbo T, Torjesen P, Urbancsek J, et al. Leptin and leptin binding activity in the preovulatory follicle of polycystic ovary syndrome patients. Scand J Clin Lab Invest 2000;60:649-55.

4. Acet M, Celik N, Acet T, Ilhan S, Yardim M, Aktun HL, et al. Serum and follicular fluid irisin levels in poor and high responder women undergoing IVF/ICSI. Eur Rev Med Pharmacol Sci 2016;20: 1940-6.

5. Celik O, Aydin S, Celik N, Yilmaz M. Peptides: basic determinants of reproductive functions. Peptides 2015;72:34-43.

6. Celik O, Celik N, Aydin S, Aygun BK, Haberal ET, Kuloglu T, et al. Ghrelin action on GnRH neurons and pituitary gonadotropes might be mediated by GnIH-GPR147 system. Horm Mol Biol Clin Investig 2016;25:121-8.

7. Kawwass JF, Summer R, Kallen CB. Direct effects of leptin and adiponectin on peripheral reproductive tissues: a critical review. Mol Hum Reprod 2015;21:617-32.

8. Yi P, Park JS, Melton DA. Betatrophin: a hormone that controls pancreatic $\beta$ cell proliferation [retracted in: Cell 2017;168:326]. Cell 2013;153:747-58.

9. Zhang R. Lipasin, a novel nutritionally-regulated liver-enriched factor that regulates serum triglyceride levels. Biochem Biophys Res Commun 2012;424:786-92.

10. Zhu JZ, Yu CH, Li YM. Betatrophin provides a new insight into diabetes treatment and lipid metabolism (review). Biomed Rep 2014;2:447-51.

11. Ehrmann DA. Polycystic ovary syndrome. N Engl J Med 2005; 352:1223-36.

12. Calan M, Yilmaz O, Kume T, Unal Kocabas G, Yesil Senses P, Senses YM, et al. Elevated circulating levels of betatrophin are associated with polycystic ovary syndrome. Endocrine 2016;53:271-9.

13. Espes D, Lau J, Carlsson PO. Increased circulating levels of betatrophin in individuals with long-standing type 1 diabetes. Diabetologia 2014;57:50-3.

14. Chen X, Lu P, He W, Zhang J, Liu L, Yang Y, et al. Circulating betatrophin levels are increased in patients with type 2 diabetes 
and associated with insulin resistance. J Clin Endocrinol Metab 2015;100:E96-100.

15. Gomez-Ambrosi J, Pascual E, Catalan V, Rodriguez A, Ramirez B, Silva $C$, et al. Circulating betatrophin concentrations are decreased in human obesity and type 2 diabetes. J Clin Endocrinol Metab 2014;99:E2004-9.

16. Fenzl A, Itariu BK, Kosi L, Fritzer-Szekeres M, Kautzky-Willer A, Stulnig TM, et al. Circulating betatrophin correlates with atherogenic lipid profiles but not with glucose and insulin levels in insulin-resistant individuals. Diabetologia 2014;57:1204-8.

17. Slemmon JR, Blacher R, Danho W, Hempstead JL, Morgan Jl. Isolation and sequencing of two cerebellum-specific peptides. Proc Natl Acad Sci U S A 1984;81:6866-70.

18. Satoh F, Takahashi K, Murakami O, Totsune K, Ohneda M, Mizuno $Y$, et al. Cerebellin and cerebellin mRNA in the human brain, adrenal glands and the tumour tissues of adrenal tumour, ganglioneuroblastoma and neuroblastoma. J Endocrinol 1997;154:2734.

19. Pang Z, Zuo J, Morgan Jl. Cbln3, a novel member of the precerebellin family that binds specifically to Cbln1. J Neurosci 2000;20: 6333-9.

20. Matsuda K, Miura E, Miyazaki T, Kakegawa W, Emi K, Narumi S, et al. Cbln1 is a ligand for an orphan glutamate receptor delta2, a bidirectional synapse organizer. Science 2010;328:363-8.

21. Mazzocchi G, Andreis PG, De Caro R, Aragona F, Gottardo L, Nussdorfer GG. Cerebellin enhances in vitro secretory activity of human adrenal gland. J Clin Endocrinol Metab 1999;84:632-5.

22. Albertin G, Malendowicz LK, Macchi C, Markowska A, Nussdorfer GG. Cerebellin stimulates the secretory activity of the rat adrenal gland: in vitro and in vivo studies. Neuropeptides 2000;34:7-11.

23. Burnet PW, Bretherton-Watt D, Ghatei MA, Bloom SR. Cerebellinlike peptide: tissue distribution in rat and guinea-pig and its release from rat cerebellum, hypothalamus and cerebellar synaptosomes in vitro. Neuroscience 1988;25:605-12.

24. Strowski MZ, Kaczmarek P, Mergler S, Wiedenmann B, Domin D, Szwajkowski P, et al. Insulinostatic activity of cerebellin: evidence from in vivo and in vitro studies in rats. Regul Pept 2009;157:1924.

25. Rotterdam ESHRE/ASRM-Sponsored PCOS Consensus Workshop
Group. Revised 2003 consensus on diagnostic criteria and longterm health risks related to polycystic ovary syndrome. Fertil Steril 2004;81:19-25.

26. Matthews DR, Hosker JP, Rudenski AS, Naylor BA, Treacher DF, Turner RC. Homeostasis model assessment: insulin resistance and beta-cell function from fasting plasma glucose and insulin concentrations in man. Diabetologia 1985;28:412-9.

27. Aydin S. A short history, principles, and types of ELISA, and our laboratory experience with peptide/protein analyses using ELISA. Peptides 2015;72:4-15.

28. Crosignani PG, Ragni G, Parazzini F, Wyssling H, Lombroso G, Perotti L. Anthropometric indicators and response to gonadotrophin for ovulation induction. Hum Reprod 1994;9:420-3.

29. Wass P, Waldenstrom U, Rossner S, Hellberg D. An android body fat distribution in females impairs the pregnancy rate of in-vitro fertilization-embryo transfer. Hum Reprod 1997;12:2057-60.

30. Wang JX, Davies MJ, Norman RJ. Polycystic ovarian syndrome and the risk of spontaneous abortion following assisted reproductive technology treatment. Hum Reprod 2001;16:2606-9.

31. Agarwal SK, Vogel K, Weitsman SR, Magoffin DA. Leptin antagonizes the insulin-like growth factor-I augmentation of steroidogenesis in granulosa and theca cells of the human ovary. J Clin Endocrinol Metab 1999;84:1072-6.

32. Fedorcsak P, Dale PO, Storeng R, Tanbo T, Abyholm T. The impact of obesity and insulin resistance on the outcome of IVF or ICSI in women with polycystic ovarian syndrome. Hum Reprod 2001; 16:1086-91.

33. Stadtmauer LA, Toma SK, Riehl RM, Talbert LM. Metformin treatment of patients with polycystic ovary syndrome undergoing in vitro fertilization improves outcomes and is associated with modulation of the insulin-like growth factors. Fertil Steril 2001; 75:505-9.

34. Celik O, Yesilada E, Hascalik S, Celik N, Sahin I, Keskin L, et al. Angiotensin-converting enzyme gene polymorphism and risk of insulin resistance in PCOS. Reprod Biomed Online 2010;20:492-8.

35. Gonzalez F, Sia CL, Shepard MK, Rote NS, Minium J. Inflammation in response to glucose ingestion is independent of excess abdominal adiposity in normal-weight women with polycystic ovary syndrome. J Clin Endocrinol Metab 2012;97:4071-9. 